\title{
Method for reconstructing the real coordinates of an object from its plane image
}

\author{
V N Nesterov ${ }^{1,2}$, V M Mukhin ${ }^{1}$ and D V Nesterov ${ }^{1}$ \\ ${ }^{1}$ JSC «Samara Electromechanical Plant», Stepan Razin street, 16, Samara, Russia, 443099 \\ ${ }^{2}$ Samara National Research University, Moskovskoe shosse, 34A, Samara, Russia, 443086 \\ e-mail: nesterov.ntc@gmail.com
}

\begin{abstract}
Original method of reconstructing the real coordinates of moving objects from their plane images is presented. The method uses multidimensional test objects whose parameters are measures and which are known with high accuracy. In order to ensure the process of measuring movements in real space a test object must be connected with a real object and mathematical models of images of multicomponent movements of a test object must be formed. The parameters of test objects that are used in the named mathematical models are vectors. Such models are used in the construction of systems of measurement equations the solution of which gives the desired components movements of a moving object in 3D space. The method was experimentally tested on specially created stand.
\end{abstract}

\section{Introduction}

Problem of the impossibility of recovering the real coordinates of objects in three-dimensional space from their plane image is one of the most serious problems in robotics. This problem was discussed in the fundamental work of well-known experts in the field of robotics and technical vision $\mathrm{K}$. Fu, R. González and K. Li [1]. A more modern work provides a broad overview of various approaches that are used to solve this ill-posed problem [2]. Binocular vision is traditionally used to solve this problem in robotics and other areas. However, this solution leads to additional problems concerning the calibration of cameras, their placement in space, special algorithms for processing the received video information, etc. If you look at the problem more broadly, you should mention the works related to geo-informatics [3] or architecture [4]. Currently, geographic information systems are increasingly complemented by tools for working with spatial geometric objects, which allows solving problems of 3D modeling of objects that are spatially referenced to the surface of the Earth. The reconstruction of 3D coordinates of objects from plane projections in photogrammetry is solved by processing stereo pairs of images [5]. To illustrate the problem consider the process of imaging a 3D object on the surface of a video camera image. With some simplifications the imaging circuit is shown on the figure 1.

On the figure 1 the image plane coincides with the plane $x, y$ of the video camera's coordinate system $\{0, \mathbf{x}, \mathbf{y}, \mathbf{z}\}$, and the optical axis, which is set in the center of the lens, is directed along the axis $z$ The center of the image plane is the origin, and the center of the lens has coordinates $(0,0, \lambda)$. 
Denote the coordinates of a point in 3D space located at a distance in front of the lens, as $(X, Y, Z)$. For all points of controlled space: $Z>\lambda$. From consideration of the corresponding right-angled triangles on the figure 1 , we can write:

$$
\frac{x_{0}}{\lambda}=\frac{X_{1}}{\lambda-Z_{1}}, \quad \frac{y_{0}}{\lambda}=\frac{Y_{1}}{\lambda-Z_{1}} .
$$

Then the relationship between the coordinates of points $M_{1}\left(X_{1}, Y_{1}, Z_{1}\right)$ and $m\left(x_{0}, y_{0}\right)$ is determined by the functions:

$$
x_{0}=\frac{\lambda X_{1}}{\lambda-Z_{1}}, \quad y_{0}=\frac{\lambda Y_{1}}{\lambda-Z_{1}} .
$$

They can be used to go from the Cartesian coordinates of a point $M_{1}\left(X_{1}, Y_{1}, Z_{1}\right)$ to the coordinates of a point of its image $m\left(x_{0}, y_{0}\right)$.

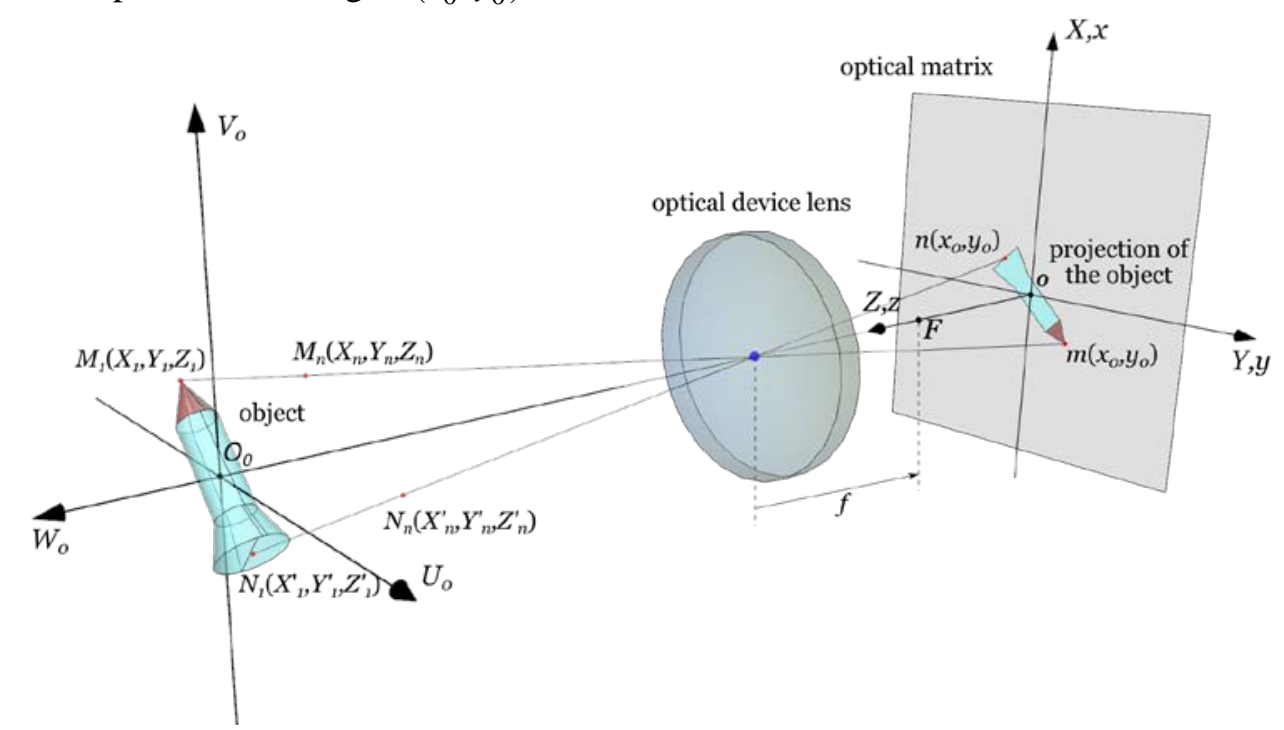

Figure 1. Image formation scheme.

Suppose that the point specified in the image has coordinates $\left(x_{0}, y_{0}, 0\right)$. In this case, the entire image plane on the $z$ axis has a coordinate $z=0$. The point of the image $m\left(x_{0}, y_{0}\right)$ corresponds to all points lying on a line that passes through the points with coordinates $\left(x_{0}, y_{0}, 0\right)$ and $(0,0, \lambda)$. The equations of this line in Cartesian coordinates are:

$$
X_{1}=\frac{x_{0}}{\lambda}\left(\lambda-Z_{1}\right), \quad Y_{1}=\frac{y_{0}}{\lambda}\left(\lambda-Z_{1}\right) .
$$

The ambiguity of this transformation is obvious. It is impossible to uniquely reconstruct a point $M_{1}\left(X_{1}, Y_{1}, Z_{1}\right)$ in 3D space by the coordinates of its image $m\left(x_{0}, y_{0}\right)$.

In all known methods for solving this ill-posed problem, including in the above-mentioned works, information redundancy is organized, for example, by using an additional camera (binocular vision) or using another method of obtaining at least two projections of a real three-dimensional object.

To solve this problem in recent years, the authors develop an original scientific direction, called: the method of multidimensional test objects in optical measurement systems [6].

To ensure the measurement process, a test object must be connected with a real object. And test object must have known geometrical parameters that are used as measures [7, 8]. At the same time, mathematical models of images of multicomponent movements are formed, which contain the parameters of test objects. These parameters are included in the models in the form of vectors and they are called tests. This is the basis of the method. Next we look at the basics of this method in more detail. 


\section{The main points of the multidimensional test objects method}

Note that the following provisions and models have a specific practical orientation and, above all, relate to the restoration of informative movements of 3D objects in space. Therefore, it should be borne in mind certain restrictions on the subject area, although according to the authors' considerations, the provisions of the method can be extended to a wider range of tasks.

Since we are talking about the reconstruction of the components of the multidimensional movement of objects in three-dimensional space, which are vector quantities, the elements of multidimensional test objects included in the models are also vector quantities. A feature of the method is that the parameters of a multidimensional test object reflect the multidimensionality of will be measured movements and are functionally associated with them in the models in the process of forming the corresponding measurement and computational algorithms:

$$
\left.\begin{array}{r}
\mathbf{X}_{x}(\mathbf{r}, \tau)=\mathbf{F}\left(\mathbf{x}_{1 x}(\mathbf{r}, \tau), \ldots, \mathbf{x}_{p x}(\mathbf{r}, \tau), \mathbf{L}_{1 x}, \ldots, \mathbf{L}_{q x}\right) ; \\
\mathbf{X}_{y}(\mathbf{r}, \tau)=\mathbf{F}\left(\mathbf{x}_{1 y}(\mathbf{r}, \tau), \ldots, \mathbf{x}_{p y}(\mathbf{r}, \tau), \mathbf{L}_{1 y}, \ldots, \mathbf{L}_{q y}\right) ; \\
\mathbf{X}_{z}(\mathbf{r}, \tau)=\mathbf{F}\left(\mathbf{x}_{1 z}(\mathbf{r}, \tau), \ldots, \mathbf{x}_{p z}(\mathbf{r}, \tau), \mathbf{L}_{1 z}, \ldots, \mathbf{L}_{q z}\right),
\end{array}\right\}
$$

where $\mathbf{X}_{x}(\mathbf{r}, \tau), \mathbf{X}_{y}(\mathbf{r}, \tau), \mathbf{X}_{z}(\mathbf{r}, \tau)$ are projections of multi-component movements on the axis of the Cartesian coordinate system; $\mathbf{r}$ is radius-vector from the beginning of the base coordinate system to the controlled point of the object under study; $\tau$ is time; $\mathbf{F}$ is components link function $\mathbf{x}_{1 k}(\mathbf{r}, \tau), \ldots, \mathbf{x}_{p k}(\mathbf{r}, \tau)$ and $\mathbf{L}_{1 k}, \ldots, \mathbf{L}_{q k} \mathbf{L}_{1 k}, \ldots, \mathbf{L}_{q k}$ of the coordinate component $\mathbf{L}_{k}(k \in\{x, y, z\})$ of the multidimensional test $\mathbf{L} ; \mathbf{x}_{1 k}(\mathbf{r}, \tau), \ldots, \mathbf{x}_{p k}(\mathbf{r}, \tau)$ are informative components of the $k$ coordinate component of the multicomponent movement $\mathbf{X}(\mathbf{r}, \tau) ; p$ is number of informative components of multicomponent movement; $\mathbf{L}_{1 k}, \ldots, \mathbf{L}_{q k}$ are components of the $k$ coordinate component $\mathbf{L}_{k}$ of the multidimensional test $\mathbf{L} ; p$ is number of components of the $k$ coordinate component $\mathbf{L}_{k}$ of the multidimensional test $\mathbf{L}$.

As already noted, the principal feature of the model (4) is the introduction of test objects $\mathbf{L}_{1 k}, \ldots, \mathbf{L}_{q k}$, which must be indicated in vector form as informative components known in it.

Figure 2 shows, as an illustration, a two-dimensional test object, which was obtained by a combination of two one-dimensional.

The test object is located in the plane $\mathrm{O}_{0} \mathrm{X}_{0} \mathrm{Y}_{0}$ and has the following parameters (tests), which are known with high accuracy:

$$
\begin{aligned}
& \mathrm{AO}_{\mathrm{i}}=n L_{A B x} \text { и } \mathrm{BO}_{\mathrm{i}}=(1-n) L_{A B x},(n=0,5) ; \\
& \mathrm{CO}_{\mathrm{i}}=n L_{C D y} \text { и } \mathrm{DO}_{\mathrm{i}}=(1-n) L_{C D y},(n=0,5) ; \\
& \mathrm{EB}=(1-n) L_{A B x} \text { и } \mathrm{FD}=(1-n) L_{C D y},(n=0,75) .
\end{aligned}
$$

The test object $\mathrm{O}_{0} \mathrm{X}_{0} \mathrm{Y}_{0}$ is a two-dimensional and multicomponent, and its parameters in models (5) are used in a vector form, for example:

$$
\begin{aligned}
& \mathbf{A O}_{\mathrm{i}}=n \mathbf{L}_{A B x}=n L_{A B x} \cdot \mathbf{i},(n=0,5) ; \mathbf{B O} \mathbf{O}_{\mathrm{i}}=(1-n) \mathbf{L}_{A B x}=(1-n) L_{A B x} \cdot \mathbf{i},(n=0,5) ; \\
& \mathbf{C O}_{\mathrm{i}}=n \mathbf{L}_{C D y}=n L_{C D y} \cdot \mathbf{j},(n=0,5) ; \mathbf{D O} \mathbf{O}_{\mathrm{i}}=(1-n) \mathbf{L}_{C D y}=(1-n) L_{C D y} \cdot \mathbf{j},(n=0,5) ; \\
& \mathbf{E B}=(1-n) \mathbf{L}_{A B x}=(1-n) L_{A B x} \cdot \mathbf{i},(n=0,75) ; \mathbf{F D}=(1-n) \mathbf{L}_{C D y}=(1-n) L_{C D y} \cdot \mathbf{j},(n=0,75),
\end{aligned}
$$

where $\mathbf{i}$ and $\mathbf{j}$ are basis vectors whose direction coincides with the direction of the axes $\mathrm{O}_{0} \mathrm{X}_{0}$ and $\mathrm{O}_{0} \mathrm{Y}_{0}$.

Here the problem is not set to classify or demonstrate the variety of test objects. Here are formulated only the basic requirements for them, which are fundamental.

Let us draw an analogy between the parameters (components) of a multidimensional test object and the components of complex displacements. Then the components of multidimensional tests or their 
projections on the coordinate axes will be considered as multicomponent quantities - multicomponent tests, the components of which are also vector quantities.

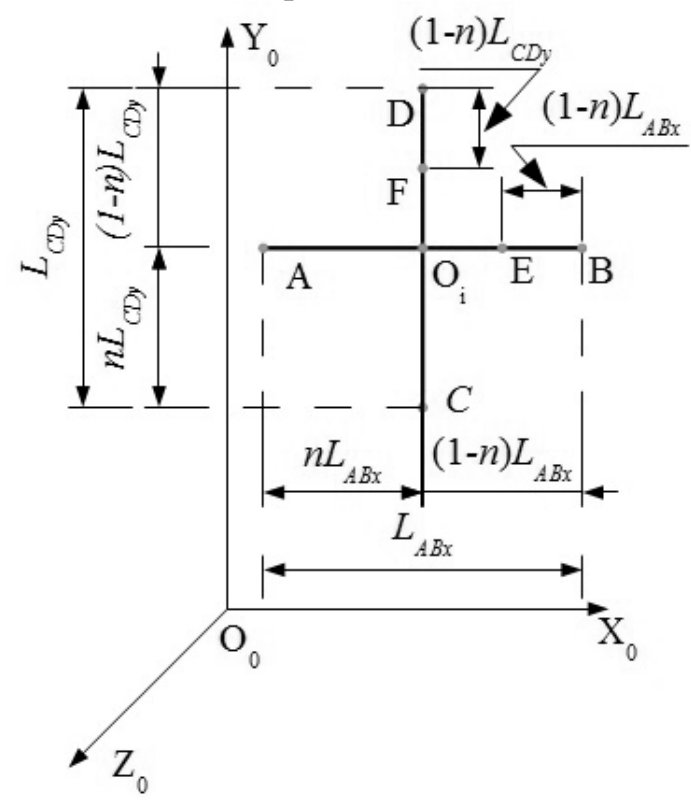

Figure 2. 2D test object ABCD.

Accordingly, the general method of forming multidimensional tests and the function of the connection of their components with multicomponent quantities fall under the basic principles of the concept of vector multicomponent physical quantities [5] and are formulated as follows:

- multidimensional multicomponent tests are considered as functions of the set of their components;

- functions of communication of the named components in models of multicomponent tests are determined by the laws of vector algebra;

- models of vector multidimensional multicomponent tests allow the multivariate representation of these components, depending on the problem being solved.

- models of vector multidimensional multicomponent tests allow to give alternative representation of these components, depending on the problem being solved.

Based on the provisions about the multidimensional test object, we define the type of function of communication $\mathbf{F}$ of informative components $\mathbf{x}_{1 k}(\mathbf{r}, \tau), \ldots, \mathbf{x}_{p k}(\mathbf{r}, \tau)$ and components $\mathbf{L}_{1 k}, \ldots, \mathbf{L}_{q k}$ of the coordinate component $\mathbf{L}_{k}$ of the multidimensional test $\mathbf{L}$ in model (4):

$$
\mathbf{F}_{i k}\left\{\mathbf{x}_{1 k}(\mathbf{r}, \tau), \ldots, \mathbf{x}_{p k}(\mathbf{r}, \tau), \mathbf{L}_{1 k}, \ldots, \mathbf{L}_{q k}\right\}=\sum_{k}^{\{x, y . z\}} \sum_{u=1}^{q} v_{i u k} \mathbf{L}_{i u k}+\sum_{k}^{\{x, y, z\}} \sum_{j=1}^{p} \eta_{i j k} \mathbf{x}_{i j k}(\mathbf{r}, \tau),
$$

where $i$ is the sequence number of the communication function; $k \in\{x, y, z\}$ is the set of coordinate components; $u$ is the serial number of the components of the multi-component test $\mathbf{L}_{i u k}$; $j$ is the sequence number of the informative components of the coordinate component of the multicomponent displacement $\mathbf{X}_{k}(\mathbf{r}, \tau) ; v_{i u k} \in[0,1]$ are the weights reflecting the absence - 0 - or the presence $(0,1]$ - the corresponding component of a multicomponent test $\mathbf{L}_{i u k}$ in model (5); $\eta_{i j k} \in[0,1]$ are the weights reflecting the absence -0 - or the presence - $(0,1]$ - the corresponding informative component $\mathbf{x}_{i j k}(\mathbf{r}, \tau)$ in model (5).

Using (5) it is possible to present model (4) in the following form: 


$$
\left.\begin{array}{l}
\mathbf{X}_{i x}(\mathbf{r}, \tau)=\sum_{u=1}^{q} v_{i u x} \mathbf{L}_{i u x}+\sum_{j=1}^{p} \eta_{i j x} \mathbf{x}_{i j x}(\mathbf{r}, \tau) ; \\
\mathbf{X}_{i y}(\mathbf{r}, \tau)=\sum_{u=1}^{q} v_{i u y} \mathbf{L}_{i u y}+\sum_{j=1}^{p} \eta_{i j y} \mathbf{x}_{i j y}(\mathbf{r}, \tau) ; \\
\mathbf{X}_{i z}(\mathbf{r}, \tau)=\sum_{u=1}^{q} v_{i u z} \mathbf{L}_{i u z}+\sum_{j=1}^{p} \eta_{i j z} \mathbf{x}_{i j z}(\mathbf{r}, \tau),
\end{array}\right\}
$$

where the vectors $\mathbf{L}_{i u x}, \mathbf{L}_{i u y}, \mathbf{L}_{i u z}, \mathbf{x}_{i j x}, \mathbf{x}_{i j y}, \mathbf{x}_{i j z}$ are defined in one-dimensional spaces that coincide with the corresponding axes of the Cartesian coordinate system.

Such a representation of model (5), while retaining its universal character, provides a mechanism for adaptation to specific practical tasks through the use of a combination of coefficients $v_{i u x} \in[0,1]$, $v_{i u y} \in[0,1], v_{i u z} \in[0,1], \eta_{i j x} \in[0,1], \eta_{i j y} \in[0,1], \eta_{i j z} \in[0,1]$ in the field of their definition.

The following is figure 3 , which illustrates the process of moving the test object, on the basis of which the model (6) was obtained. It shows the movement in space of a cruciform shape, which is a test object, and the reflection of this movement on a flat matrix of the optical instrument.

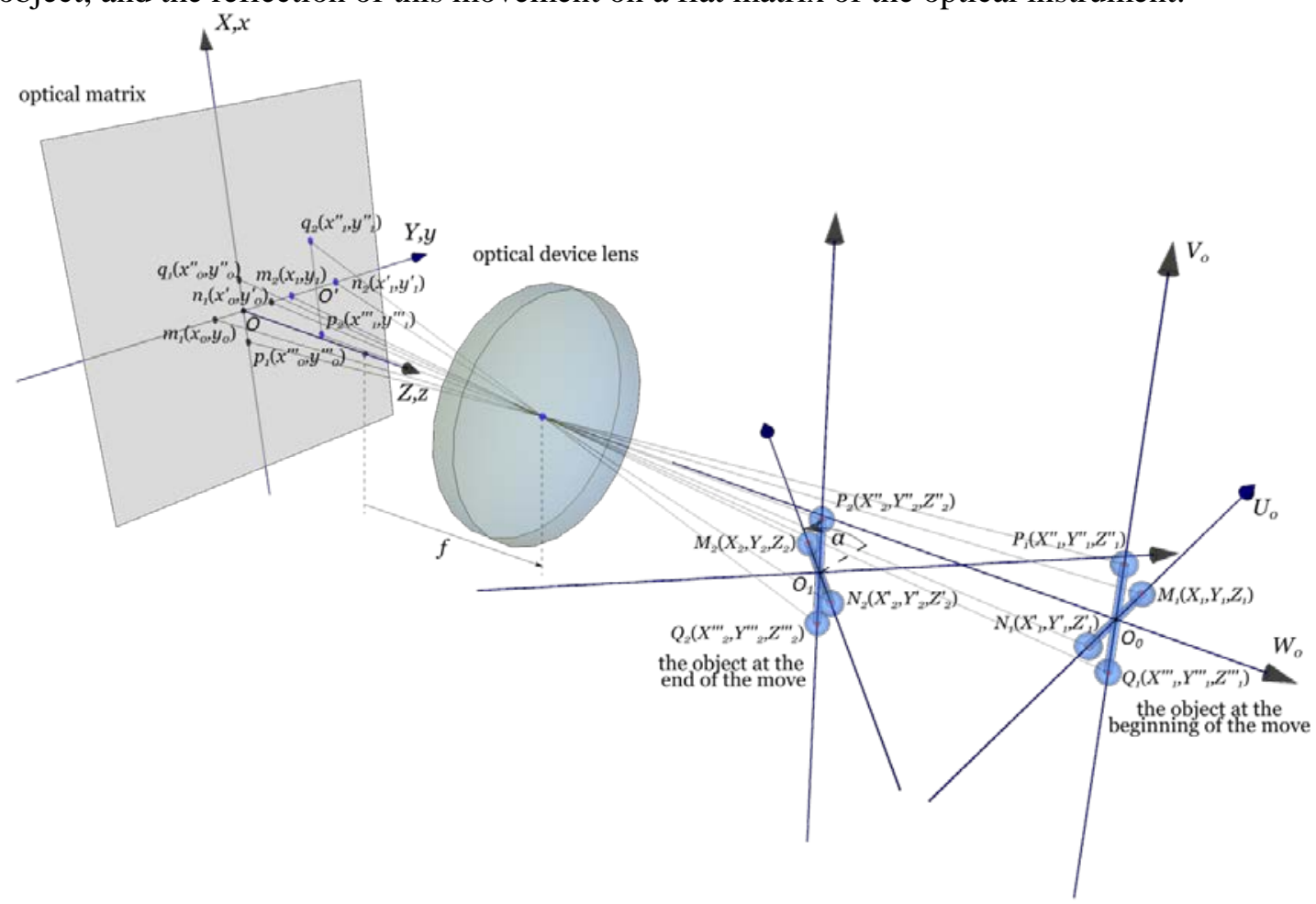

Figure 3. Illustration for model (6).

Any movement, including multicomponent, is described in accordance with the laws and provisions of vector algebra. When considering the projections of vector quantities onto the plane and introducing special agreements, it is possible to significantly simplify the process of synthesizing complex mathematical models by formally generating them, which is necessary to search for appropriate measurement and computational algorithms [9]. The information redundancy embodied in the models in the form of parameters of a multidimensional test object allows us to approach its solution.

\section{Physical realizability of the measurement method}

Based on the formulated provisions on multidimensional test objects, we list the method-forming features, the implementation of which will solve the problem of the physical realizability of the method: 
1. The presence (the ability to form) a system of $n$ equations that are asymmetric with respect to informative components $\mathbf{x}_{1 k}(\mathbf{r}, \tau), \ldots, \mathbf{x}_{p k}(\mathbf{r}, \tau)$ ( $k \in\{x, y, z\}$ is the set of coordinate components) displacements of the corresponding image points of the test object:

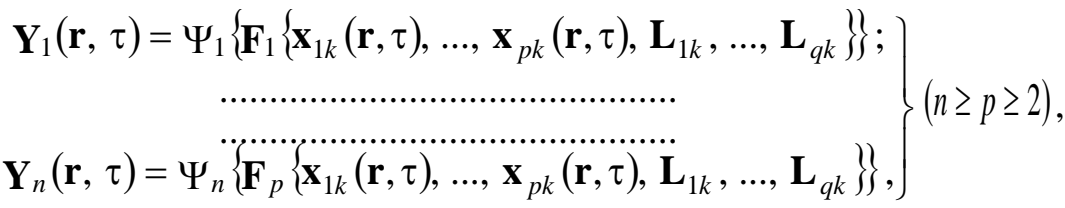

$$
\begin{aligned}
& \mathbf{F}_{1}\left\{\mathbf{x}_{1 k}(\mathbf{r}, \tau), \ldots, \mathbf{x}_{p k}(\mathbf{r}, \tau), \mathbf{L}_{1 k}, \ldots, \mathbf{L}_{q k}\right\} \neq \ldots \neq \mathbf{F}_{p}\left\{\mathbf{x}_{1 k}(\mathbf{r}, \tau), \ldots, \mathbf{x}_{p k}(\mathbf{r}, \tau), \mathbf{L}_{1 k}, \ldots, \mathbf{L}_{q k}\right\},
\end{aligned}
$$

where $\mathbf{Y}_{1}(\mathbf{r}, \tau), \ldots, \mathbf{Y}_{n}(\mathbf{r}, \tau)$ are functions of displacement of the corresponding points of the image of the object being monitored relative to the selected on the image of the points of reference; $\mathbf{F}_{1}\left\{\mathbf{x}_{1 k}(\mathbf{r}, \tau), \ldots, \mathbf{x}_{p k}(\mathbf{r}, \tau), \mathbf{L}_{1 k}, \ldots, \mathbf{L}_{q k}\right\}, \ldots, \mathbf{F}_{p}\left\{\mathbf{x}_{1 k}(\mathbf{r}, \tau), \ldots, \mathbf{x}_{p k}(\mathbf{r}, \tau), \mathbf{L}_{1 k}, \ldots, \mathbf{L}_{q k}\right\}$ are vector functions of the set of informative components $\mathbf{x}_{1 k}(\mathbf{r}, \tau), \ldots, \mathbf{x}_{p k}(\mathbf{r}, \tau)$ and components $\mathbf{L}_{1 k}, \ldots, \mathbf{L}_{q k}$ of the coordinate component $\mathbf{L}_{k}$ of a multidimensional test object (of the multidimensional test) $\mathbf{L}$.

2. The ability to realization special measuring and computing algorithms:

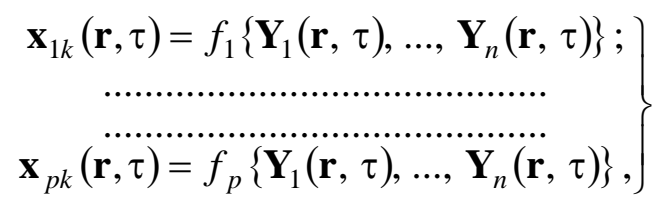

condition of the existence of which, with continuity and differentiability $\mathbf{Y}_{1}(\mathbf{r}, \tau), \ldots, \mathbf{Y}_{n}(\mathbf{r}, \tau)$ in the whole range of measurement, is the inequality of zero Jacobian:

$$
\operatorname{det}\left[\frac{\partial \mathbf{Y}_{i}(\mathbf{r}, \tau)}{\partial \mathbf{x}_{j k}(\mathbf{r}, \tau)}\right] \neq 0 \quad i=\overline{1, n}, j=\overline{1, p} .
$$

The condition (10) is ensured by the implementation of the "asymmetry" of the values $\mathbf{Y}_{1}(\mathbf{r}, \tau), \ldots, \mathbf{Y}_{n}(\mathbf{r}, \tau)$ relative to their constituent components $\mathbf{x}_{1 k}(\mathbf{r}, \tau), \ldots, \mathbf{x}_{p k}(\mathbf{r}, \tau)$ and $\mathbf{L}_{1 k}, \ldots, \mathbf{L}_{q k}$, which is expressed by the inequality (8).

Obviously, when using a single-channel optical system, the functions $\psi_{1}, \ldots, \psi_{n}$ are the same. If we use the transmission coefficient $\sigma$ of the optical converter, then the system of equations (7) can be rewritten as follows:

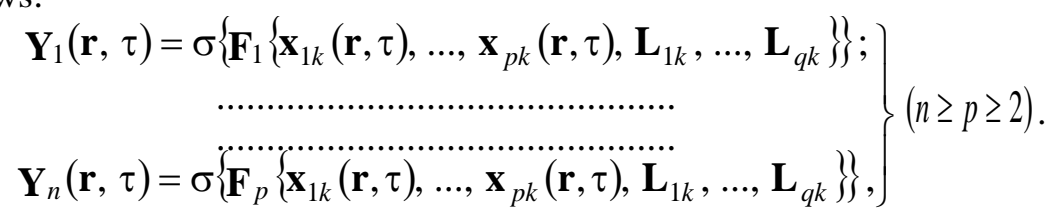

Having considered the system (11) we can understand all the perspectives of the optical measurement method. For a more detailed consideration of the implementation of the proposed method, refer to [6-9]. The originality and novelty of the measurement method is confirmed by patents of the Russian Federation for inventions [10-12]. The method was experimentally tested on specially created stand where the cruciform figure was used as a test object. The details of the experiment and its results are presented in a separate section in paper [6].

\section{Conclusions}

Summing up, we can conclude that, on the basis of the method of multidimensional test objects, the incorrect problem of reconstructing the coordinates of objects moving in three-dimensional space from their flat image has been solved. 
The basics of optical measurements of informative components of multicomponent displacements based on the method of multidimensional test objects, providing information redundancy at the input of an optical image receiver, are also presented. Additional information at the system input in the form of known parameters of multidimensional test objects creates opportunities for improving the quality and quantity of the resulting information.

Separate attention is required for the problem of generating multidimensional test objects, to investigate the effects of their type, quantity and optimization of test components (parameters of a multidimensional test object) on the quality of the measuring system both in the general theoretical aspect and for solving specific applied problems. These questions should be the subject of further research.

\section{References}

[1] Fu K, Gonzalez R and Lee K 1989 Robotics (Moscow: World) p 624

[2] Oswald M R, Toppe E, Nieuwenhuis C and Cremers D 2013 A review of geometry recovery from a single image focusing on curved object reconstruction Innovations for shape analysis: models and algorithms (Berlin Heidelberg: Springer-Verlag) 343-378 DOI: 10.1007/978-3-64234141-0

[3] Kostyuk Yu L, Paramonov A S and Gritsenko V G 1998 The technology of creating 3D models of objects on flat projections and its application in geoinformatics Geoinformatics: Theory and Practice (Tomsk: Tomsk University) 1 96-106

[4] Dzwierzynska J 2016 Reconstructing architectural environment from the perspective image World multidisciplinary civil engineering-architecture-urban planning symposium, WMCAUS (ScienceDirect: Proc. engineering) 161 1445-1451

[5] Kostyuk Yu L 2002 Efficient algorithms for processing and displaying graphic data and their implementation in software packages Abstract of thesis ... doc. tech. of science (Tomsk: Tomsk University) p 39

[6] Nesterov V N, Mukhin V M and Meshchanov A V 2013 The method of multidimensional test objects in optical measurement systems (Samara: Publishing House of the Samara Scientific Centre of the Russian Academy of Sciences) p 224

[7] Nesterov V N and Meshchanov A V 2006 Models for vector multicomponent physical quantities and a multivariate test method for optical measurement systems Measurement Techniques 49(12) 1182-1188

[8] Nesterov V N and Meshchanov A V 2007 Theoretical principles of optical measurements on the components of multicomponent displacements for mobile objects on the basis of multivariate tests Measurement Techniques 50(11) 1127-1136

[9] Nesterov V N and Zhmurov D B 2007 Formal synthesis of a data-acquisition system for multicomponent physical quantities Measurement Techniques 50(9) 903-907

[10] Nesterov V N, Mukhin V M and Meshchanov A V 2008 The way to measure the components of complex movements of an object Russian Federation patent No RU2315948 URL: http://www1.fips.ru/fips_servl/fips_servlet?DB=RUPAT\&DocNumber=2315948\&TypeFile=ht $\mathrm{ml}$

[11] Nesterov V N, Mukhin V M and Meshchanov A V 2012 The method of controlling the accuracy of contour movements of industrial robots Russian Federation patent No RU 2466858 URL: http://www1.fips.ru/fips_servl/fips_servlet?DB=RUPAT\&DocNumber=2466858\&TypeFile=ht $\mathrm{ml}$

[12] Nesterov V N, Nesterov D V and Mukhin V M 2017 The way to measure the components of complex movements of an object Russian Federation patent No RU2610425 URL: http://www1.fips.ru/fips_servl/fips_servlet?DB=RUPAT\&DocNumber=2610425\&TypeFile=ht 